\title{
Management of Staphylococcus aureus bacteremia in adults
}

\author{
Anthony D. Bai MD, Andrew M. Morris MD SM
}

Cite as: CMAJ 2019 September 3;191:E967. doi: 10.1503/cmaj.190363

\section{Cases in which a blood culture grows Staphylococcus aureus 1. should always be treated as a true bloodstream infection Staphylococcus aureus bacteremia is associated with substantial mortality and complications, including endocarditis and metastatic infection requiring specific investigations and treatment. ${ }^{1}$ Given the potential for substantial mortality and morbidity, patients with growth of $S$. aureus in blood culture should always be treated. ${ }^{1}$}

\section{2}

\section{Expert consultation is suggested for all patients with S. aureus bacteremia}

In observational and quasi-experimental studies, consultation with infectious disease specialists improved the quality of care in patients with S. aureus bacteremia, including early source control, follow-up blood culture, echocardiography, and appropriate choice and duration of antibiotic therapy. ${ }^{1}$ These measures decrease mortality and facilitate earlier discharge. ${ }^{1}$

\section{3}

Initial antibiotic therapy for S. aureus bacteremia should be

intravenous and tailored to susceptibility once known

Vancomycin can be used as empiric therapy before susceptibility is known and as definitive therapy for methicillin-resistant $S$. aureus. ${ }^{2}$ Definitive therapy for methicillin-susceptible $S$. aureus should be cefazolin or an antistaphylococcal penicillin., Evidence supporting oral antibiotic therapy is currently limited.

All patients with S. aureus bacteremia should undergo thorough evaluation for infectious source and secondary infectious foci

About $10 \%-20 \%$ of patients with S. aureus bacteremia have infective endocarditis. ${ }^{4}$ All patients with S. aureus bacteremia should undergo echocardiography, because the presence of endocarditis has therapeutic and diagnostic implications including consideration for surgery. ${ }^{4,5}$ Patients at high risk (i.e., those with embolic events, pacemakers, prior endocarditis, prosthetic valves or intravenous drug use) need transesophageal echocardiography to exclude endocarditis. ${ }^{4}$

\section{5}

Patients with S. aureus bacteremia should be treated with at least 2 weeks of antibiotic therapy

According to consensus guidelines, patients with uncomplicated $S$. aureus bacteremia (Box 1) may be treated with 2 weeks of antibiotic therapy. ${ }^{5}$ All other patients should be treated with at least 4 weeks of antibiotic therapy. ${ }^{5}$

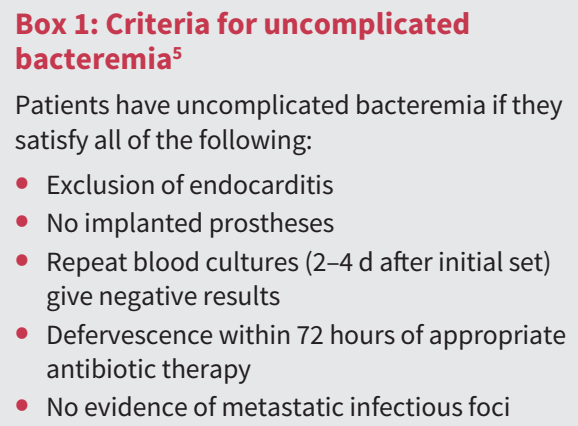

- Defervescence within 72 hours of appropriate antibiotic therapy

- No evidence of metastatic infectious foci

\section{References}

1. Vogel M, Schmitz RP, Hagel S, et al. Infectious disease consultation for Staphylococcus aureus bacteremia - a systematic review and meta-analysis. J Infect 2016;72:19-28.

2. McDanel JS, Perencevich EN, Diekema DJ, et al. Comparative effectiveness of beta-lactams versus vancomycin for treatment of methicillin-susceptible Staphylococcus aureus bloodstream infections among 122 hospitals. Clin Infect Dis 2015;61:361-7.

3. Weis S, Kesselmeier M, Davis JS, et al. Cefazolin versus antistaphylococcal penicillins for the treatment of patients with Staphylococcus aureus bacteremia. Clin Microbiol Infect 2019; 25:818-27.

4. Bai AD, Agarwal A, Steinberg M, et al. Clinical predictors and clinical prediction rules to estimate initial patient risk for infective endocarditis in Staphylococcus aureus bacteraemia: a systematic review and meta-analysis. Clin Microbiol Infect 2017;23:900-6.

5. Liu C, Bayer A, Cosgrove SE, et al.; Infectious Diseases Society of America. Clinical practice guidelines by the Infectious Diseases Society of America for the treatment of methicillin-resistant Staphylococcus aureus infections in adults and children. Clin Infect Dis 2011;52:e18-55.

\section{Competing interests: None declared.}

This article has been peer reviewed.

Affiliations: Department of Medicine (Bai), Queen's University, Kingston, Ont.; Department of Medicine (Morris), University of Toronto; Sinai Health System (Morris); University Health Network (Morris), Toronto, Ont.

Correspondence to: Andrew Morris, andrew.morris@sinaihealthsystem.ca 